\title{
Dendritic cell-based therapy for mantle cell lymphoma
}

\author{
COREY M. MUNGER, JULIE M. VOSE and SHANTARAM S. JOSHI \\ Departments of Genetics, Cell Biology and Anatomy, and Internal Medicine Section of Oncology and Hematology, \\ University of Nebraska Medical Center, Omaha, NE 68198-6395, USA
}

Received November 15, 2005; Accepted December 28, 2005

\begin{abstract}
Mantle cell lymphoma (MCL) is a B cell malignancy that is resistant to conventional therapies. High-dose therapy (HDT) followed by stem cell transplantation is effective in inducing remission. However, residual lymphoma cells are eventually responsible for the subsequent relapse. Effective therapeutic strategies to eliminate the residual lymphoma is required. In this study, we have examined the in vitro and in vivo anti-lymphoma effects of MCL-specific cytotoxic $\mathrm{T}$ lymphocytes (CTLs) that were generated using dendritic cells (DCs) fused with MCL cells for immunostimulation. Dendritic cells were generated in vitro using dendritic cellspecific medium, cytomorphology, immunophenotypes and functional capabilities of the generated DCs were studied. Such DCs were then used for the preparation of DC-MCL hybrids and the DC-MCL hybrids were used to generate CTLs against MCL cells and tested for their MCL-specific cytotoxicity in vitro and in vivo. The CTLs demonstrated MCL-specific cytotoxicity in vitro against GRANT-519, a human MCL cell line. These CTLs did not show significant effect against an irrelevant target. To test the in vivo therapeutic effect of DC-MCL hybrid-stimulated CTLs, a preclinical model consisting of NOD-SCID mice bearing Granta 519 was developed. The NOD-SCID mice bearing Granta-519 MCL tumors were treated with DC-MCL hybrids and the same donor $\mathrm{T}$ lymphocytes. There was an increase in survival $(60 \%$ in mice treated with DC-MCL hybrid approach compared to $20 \%$ in the untreated group). Histological analysis of liver from control and treated mice displayed a decrease in the number of the tumor nodules in the treatment group. These results indicate the potential of DCbased therapy for the treatment of MCL.
\end{abstract}

\section{Introduction}

Mantle cell lymphoma (MCL) is a B cell malignancy that represents approximately $6 \%$ of all non-Hodgkin's lymphomas.

Correspondence to: Professor Shantaram S. Joshi, Department of Genetics, Cell Biology, and Anatomy, University of Nebraska Medical Center, 986395 Nebraska Medical Center, Omaha, NE 68198-6395, USA

E-mail: ssjoshi@unmc.edu

Key words: dendritic cells, antigen priming, cytotoxic T lymphocytes, mantle cell lymphoma
The malignant cells are believed to originate from a subset of $\mathrm{B}$ cells in the mantle zone of secondary follicles. MCL-specific B cells display CD5, CD19, CD20, CD22, CD43, Bcl-2, and CD1 (2). In the majority of the MCL patients, a typical chromosomal translocation $\mathrm{t}(11 ; 14)(\mathrm{q} 13 ; \mathrm{q} 32)$ resulting in fusion of BCL1 locus on chromosome 11q13 and the IgH heavy chain joining-region on chromosome $14 \mathrm{q} 32$ (4) is seen. This genomic aberration results in the upregulation of Bcl-1/PRAD-1 and increased expression of cyclin D1 in the majority of MCL cases (3-5). The aggressive nature of MCL coupled with a typically short remission period, presents a significant challenge for the development of effective therapeutic options. Patients with MCL typically present with advanced disease and extranodal involvement is common in tissue such as bone marrow, liver, spleen, and GI tract (1).

Current combinatorial treatment modalities for MCL generally include induction therapy, followed by mobilization chemotherapy, and ending with high-dose therapy in conjunction with stem cell transplantation (SCT) (6-12). Combination therapy is effective at inducing remission, yet therapy-resistant residual lymphoma cells are eventually responsible for relapse and subsequent decrease in overall survival. Since MCL is resistant to most conventional therapies, new and novel treatments are needed.

Dendritic cell (DC)-based immunotherapy might be a promising strategy for treating residual MCL. Dendritic cells are effective antigen presenting cells that are capable of processing exogenous and endogenous antigens for presentation via both MHC class I and class II pathways (13-15). Taking advantage of dendritic cell biology, DCs can be loaded with tumor cell-specific antigens for the development of tumor-specific cytotoxic T lymphocytes. Specifically, DCs can be fused with MCL cells to produce immunostimulatory heterokaryons (DC-MCL hybrids) (16-22). In many ways, heterokaryons may be better compared to using specific antigenic peptides due to the sheer number of tumorspecific molecules available for the DCs to sample. The use of DC-MCL hybrids may involve multiple antigenic peptides that may stimulate the activation and production of numerous cytotoxic $\mathrm{T}$ cell clones against multiple tumor-specific antigens which can improve the overall cytotoxic response against MCL. In addition, DC-MCL fusion may also provide access to sequestered proteins previously inaccessible to DC uptake (23-25).

In this study, we investigated the generation of DC-MCL hybrids for immunostimulation of MCL-specific CTLs in vitro. In addition, we developed a NOD-SCID preclinical 
model for human MCL and demonstrated the therapeutic effects of DC-MCL hybrids and the same donor-effector $\mathrm{T}$ cells against MCL grown in NOD-SCID mice.

\section{Materials and methods}

Cell lines. The Granta 519 mantle cell lymphoma cell line was purchased from Deutsche Sammlung von Mikroorganismen und Zellkulturen (DSMZ, Germany). Granta 519 cells were maintained in DMEM (4.5 g/l glucose) containing 10\% fetal bovine serum (FBS), $2 \mathrm{mM}$ L-Glutamine, and 100 units $/ \mathrm{ml}$ penicillin, and $100 \mu \mathrm{g} / \mathrm{ml}$ streptomycin.

Mice. Female NOD/SCID mice (6-8-week-old) were purchased from Jackson Laboratories (Bar Harbor, Maine). All mice were housed and maintained in the animal care facility, with each experiment done using a protocol approved by the University of Nebraska Medical Center Institutional Animal Care and Use Committee (IACUC) guidelines.

Normal donor peripheral blood mononuclear cells. Generation of DCs from peripheral blood mononuclear cells (PBMC) was done using a modified version of the method outlined by Grunebach et al (26). Briefly, PBMCs from normal donors were harvested using a Cobe Spectra aphaeresis machine. Isolated PBCs were further purified using lymphocyte separation media with subsequent centrifugation. The mononuclear cells at the interphase were collected, washed twice in DPBS, and counted for use as a source of DCs and cytotoxic effector cells. Plastic adherence was subsequently used to separate DC precursors from the bulk mononuclear cells. Briefly, PBCs were incubated for $2.5 \mathrm{~h}$ in adherent tissue culture flasks to promote DC precursor cell adhesion. The non-adherent fraction containing predominantly $\mathrm{T}$ cells was cultured in medium containing IL-2 $(50 \mathrm{U} / \mathrm{ml})$ and used as effector cells.

Dendritic cell preparation. To generate DCs, the adherent mononuclear cells were cultured in AIM V serum-free media (Gibco) supplemented with $800 \mathrm{U} / \mathrm{ml}$ human recombinant GM-CSF (Immunex Corporation, Seattle, WA) and $300 \mathrm{U} / \mathrm{ml}$ IL-4 (BD Pharmingen, San Diego, CA) for 6 days. Half of the media was replaced on day three. After 6 days in culture, the immature DCs were placed into a maturation media consisting of serum-free AIM V supplemented with the following components: GM-CSF (800 U/ml), human IL-4 (300 U/ml), and TNF- $\alpha$ (50 ng/ml) (R\&D; Minneapolis, MN). The mature DCs were harvested on day eight for use in CTL generation.

Immunophenotypic analysis of dendritic cells. The surface phenotype of the mature DCs was assayed using a Becton Dickinson FASCStar Plus flow cytometer using the method described by Wang et al (27). Briefly, 200,000 cells were suspended in $100 \mu 1$ of $1 \mathrm{X}$ PBS containing $2 \%$ FBS in a 96-well round-bottom plate. The cells were incubated on ice for 30 min with $5 \mu 1$ of phycoerythrin (PE)-labeled antibody-specific for the following cell surface markers: CD1a, CD3, CD40, CD80, and CD86 (BD Pharmingen). After the incubation, the cells were washed 3 times with
$100 \mu 1$ PBS and resuspended in $350 \mu 1$ of $4 \%$ formalin PBS for analysis.

Functional properties of dendritic cells. The DCs were assayed for their ability to stimulate an immune response using an allogenic mixed lymphocyte reaction (allo-MLR) as described previously (27). Mononuclear cells isolated from two different normal donors were combined and plated in a 96-well flatbottom plate at a 1:1 ratio with a concentration of $10^{5}$ cells/ well. Dendritic cells were added into the culture at $5 \%$ of the total cell concentration. Control wells contained $10^{5}$ cells from each normal sample in addition to a mixture of the donor cells without DCs. The cells were incubated for 5 days at $37^{\circ} \mathrm{C}$ in $5 \% \mathrm{CO}_{2}$. On the fifth day of the incubation, $1 \mu \mathrm{Ci}$ of radiolabeled ${ }^{3} \mathrm{H}$-thymidine was added to each well and the cells were incubated an additional $18 \mathrm{~h}$. The cells were then harvested onto glass fiber disks using a PHD cell harvester (Cambridge Technologies, Watertown, MA). The filter disks were allowed to dry and were placed into scintillation vials containing $1 \mathrm{ml}$ of scintillation fluid. A liquid scintillation counter (TRI-CARB 2100 TR, Packard Instruments, Chicago, IL) was used to measure the ${ }^{3} \mathrm{H}$-thymidine incorporation.

Effector cell preparation. The non-adherent $\mathrm{T}$ cell rich mononuclear cells were cultured in serum-free AIM V media supplemented with 50 units $/ \mathrm{ml}$ of recombinant human IL-2 (Chiron, Emeryville, CA). Every three days, half of the media was replaced with a subsequent addition of fresh IL-2 $(50 \mathrm{U} / \mathrm{ml})$. On day seven, the cells were harvested for use in CTL generation.

DC-MCL fusion. Dendritic and Granta 519 cells were fused via electrofusion using a BioRad GenePulser II from a modified protocol outlined by Scott-Taylor et al (19). Briefly, the cells were washed in DPBS and mixed at a ratio of 1:5 in fusion media ( $5 \%$ glucose, DPBS), with a final cell count totaling $5 \times 10^{7}$ cells $/ \mathrm{ml}$. The cell mixture was placed into a $0.4 \mathrm{~cm}$ electroporation cuvette (BioRad) and the cells were aligned with a $4 \mathrm{sec}$ alignment pulse at $20 \mathrm{~V}$. Immediately following the alignment pulse, the cells were fused with a single pulse of $300 \mathrm{~V}$ and $24 \mu \mathrm{F}$ with the gene pulser apparatus. The fusion products were left at room temperature for $5 \mathrm{~min}$, followed by a wash in DPBS and subsequent incubation in DC maturation media. To determine fusion efficiency, a portion of the DCs and Granta 519 cells were stained red and green (Molecular Probes, Eugene, OR) respectively, prior to fusion. Following the fusion process, the hybrid cells were examined using confocal microscopy. Due to the presence of both red and green dyes, hybrid DC/MCL cells appear yellow under confocal microscopy.

Generation of MCL-specific CTLs. To generate MCL-specific CTLs, the IL-2 stimulated T-cell-enriched mononuclear cells were plated with DC-MCL primed DCs at a ratio of 1:10. The cultures were re-stimulated weekly (3 total) with DC-MCL hybrids with fresh media and IL-2 $(50 \mathrm{U} / \mathrm{ml})$ added every 2 days. The MCL-specific cytotoxicity was measured 7 days after the last restimulation. The antigen-specific CTLs and ${ }^{51}$ Cr-labeled Granta 519 or similarly labeled MDA 231 human breast cancer cells were mixed to obtain effector-to-target 
ratios (E:T) of 5:1, 12.5:1, 25:1, and 50:1 in $200 \mu \mathrm{l} /$ well. The 96-well plate was incubated at $37^{\circ} \mathrm{C}$ for $4 \mathrm{~h}$. After the incubation, the plate was centrifuged for $8 \mathrm{~min}$ at $900 \mathrm{rpm}$, followed by the removal of $140 \mu 1$ from each well to be assayed for radioactivity using a Beckman 5500 gamma counter. The percentage of lysed target cells was calculated using the following formula:

$$
\% \text { cytotoxicity }=\frac{\text { experimental } \mathrm{cpm}-\text { spontaneous } \mathrm{cpm}}{\text { total } \mathrm{cpm}-\text { spontaneous } \mathrm{cpm}}
$$

where the experimental cpm is effector + target cells, spontaneous cpm is target cells + media, and total release is target cells $+1 \%$ Triton $\mathrm{X}-100$.

Establishment of MCL animal model. Prior to initiating in vivo studies using DC DC-MCL heterokaryon-based immunotherapy, MCL tumor growth was established in NOD/SCID mice. In order to effectively establish MCL growth in the mice, $10 \times 10^{6}$ Granta 519 cells were transplanted intravenously. Three weeks post tumor transplantation, the mice were sacrificed and autopsied to examine for the presence of tumor growth using histological sections of different organs and tumor tissue from the tumor-bearing animals. Initially hematoxylin and eosin staining was also used to determine the presence of MCL cells in histological sections from organs. Immunohistochemistry, using peroxidase-conjugated antiCD45 and anti-CD19 antibodies, was used to determine the human origin of tumors within the mouse organs.

In vivo therapeutic studies. NOD/SCID mice were injected intravenously with $2 \times 10^{5}$ Granta 519 cells. Twenty-four hours prior to injection, the mice were exposed to 400 rads to sufficiently eliminate any systemic immune cells present within the mice. The mice were split into two groups (control and treatment), each consisting of five mice. Approximately $48 \mathrm{~h}$ after tumor challenge, the treatment group was treated intravenously with irradiated (1700 rads) DC/MCL hybrids along with autologous $\mathrm{T}$ cells at a concentration of $5 \times 10^{6} \mathrm{~T}$ cells and $5 \times 10^{4}$ hybrids per mouse. Treatment was administered weekly. After 4 weeks, the mice were sacrificed and spleen, liver, lung, and kidneys were removed and preserved in $10 \%$ formalin for use in histological analysis. The human origin of the tumor was confirmed as described above. Number of tumor nodules in liver was enumerated.

\section{Results}

Generation and characterization of functional and phenotypic dendritic cells. For use in the in vitro and in vivo aspects of the experiment, dendritic cells were generated and matured as previously outlined in Materials and methods. The cytomorphology, immunophenotype and functional capabilities of these DCs are shown in Fig. 1. Cytomorphological analysis of day seven DCs revealed a significant number of dendritic cells, each displaying typical DC cytomorphology (Fig. 1A). The phenotypes of the DCs were determined with flow cytometry using antibodies to DC-specific cell surface markers. The results indicated a typical mature dendritic cell phenotype, with elevated expression of CD80, CD83, and CD86 as shown

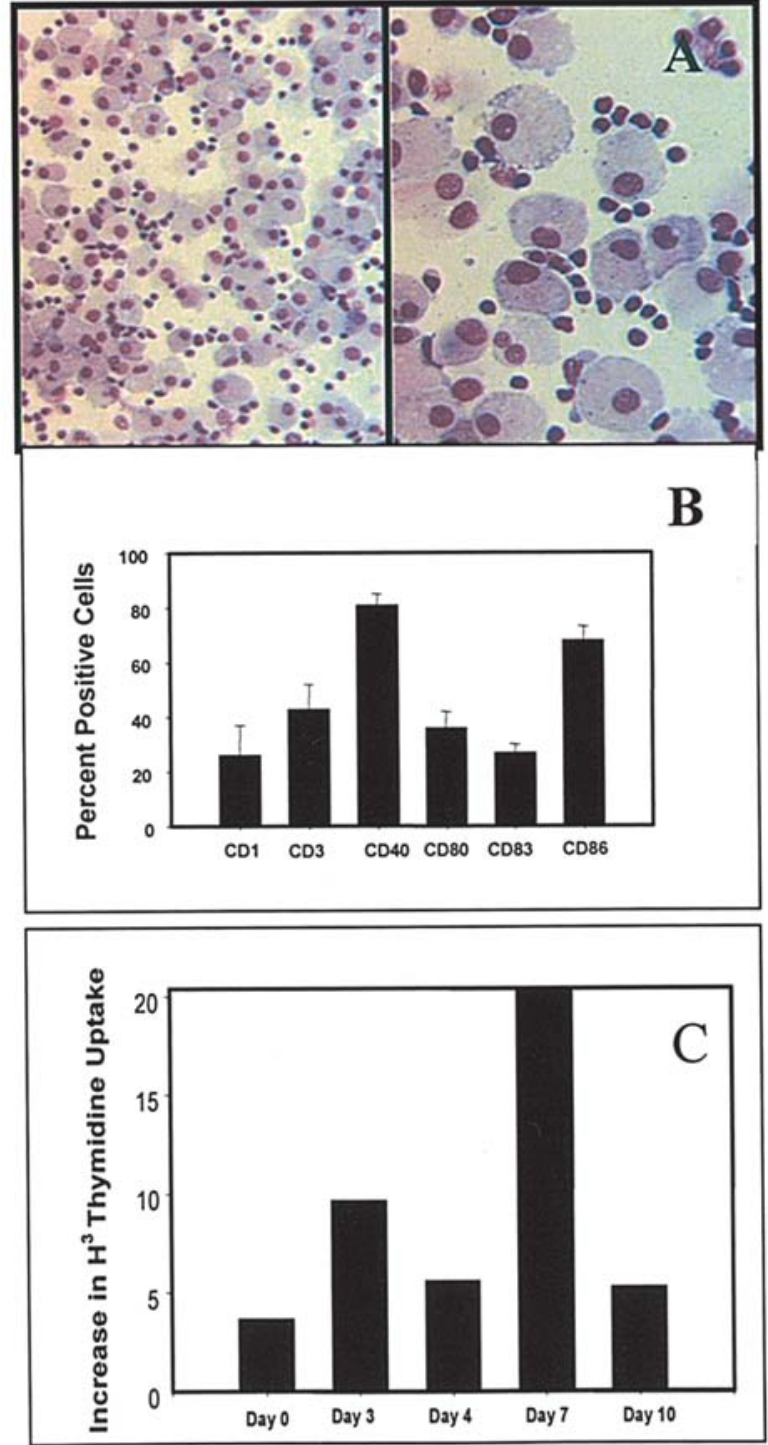

Figure 1. Characterization of DCs for their cytomorphology, immunophenotype and immunostimulatory functions. (A) The cytomorphology of dendritic cells grown in ex vivo 10 supplemented media for 7 days. The light purple, larger cells are the dendritic cells and the dark purple cells are T cells (magnification x500). (B) Phenotypic analysis of in vitro generated dendritic cells. Cells were stained with flurochrome-labeled-specific antibodies and the percent positive cells for each marker was determined using flow cytometry. The figure represents the mean \pm standard error of the mean percent positive cells for each phenotypic marker. (C) Immunostimulatory properties of cultured dendritic cells as determined using allogenic-MLR. Mononuclear cells from two different donors were cultured in vitro for 5 days with or without $5 \%$ dendritic cells. The proliferation of cells in the mixture was determined using radioactive thymidine.

in Fig. 1B. The overall expression level of the DC markers can be seen in Fig. 1B. The functional capabilities of the dendritic cells were determined using an allo-MLR assay as demonstrated in Fig. 1C. The addition of $5 \%$ DCs produced a proliferative increase in the allogenic mixed lymphocyte reaction reflecting the potent immunostimulatory properties of the DCs produced and used in these experiments. These results also demonstrated that the DCs from day 7 culture are the best for immunostimulatory effects. The results are presented as fold increase compared to control without DCs. 


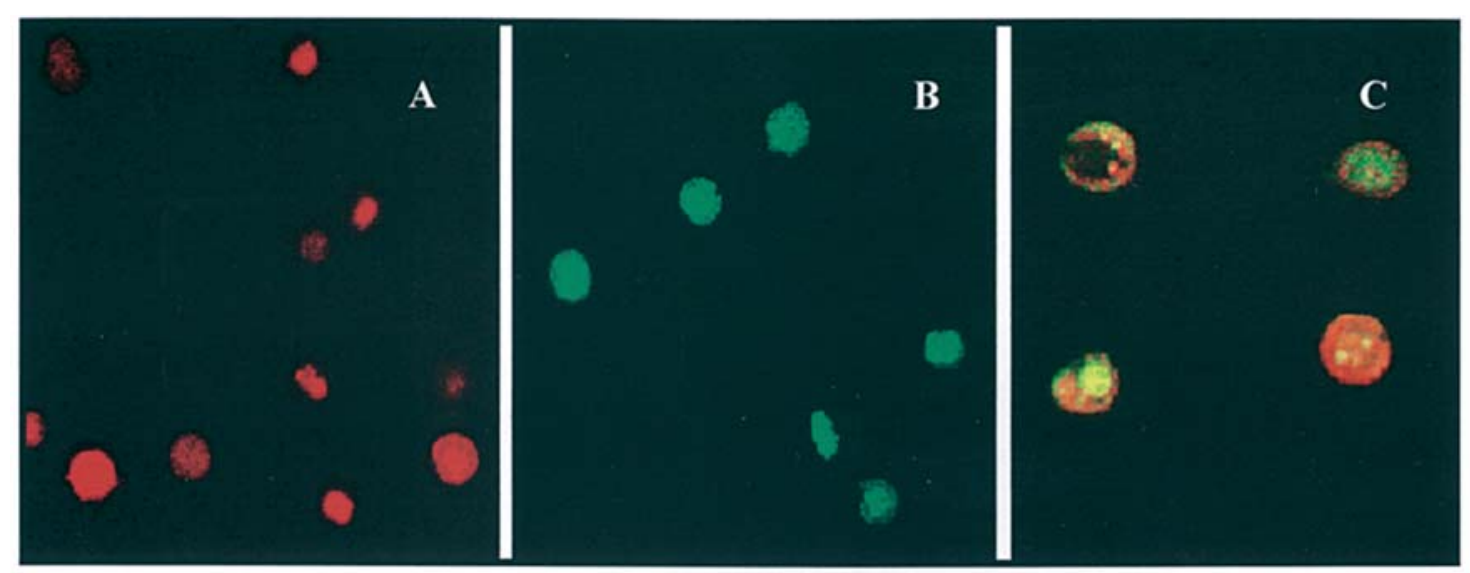

Figure 2. Determination of fusion efficiency by confocal microscopy. Each individual type of cells were stained with a particular intracellular dye prior to the fusion process: (A) dendritic cells (red), (B) Granta $519 \mathrm{MCL}$ (green), and (C) DC/MCL heterokaryons (yellow, result of fusion between DC and MCL cells).

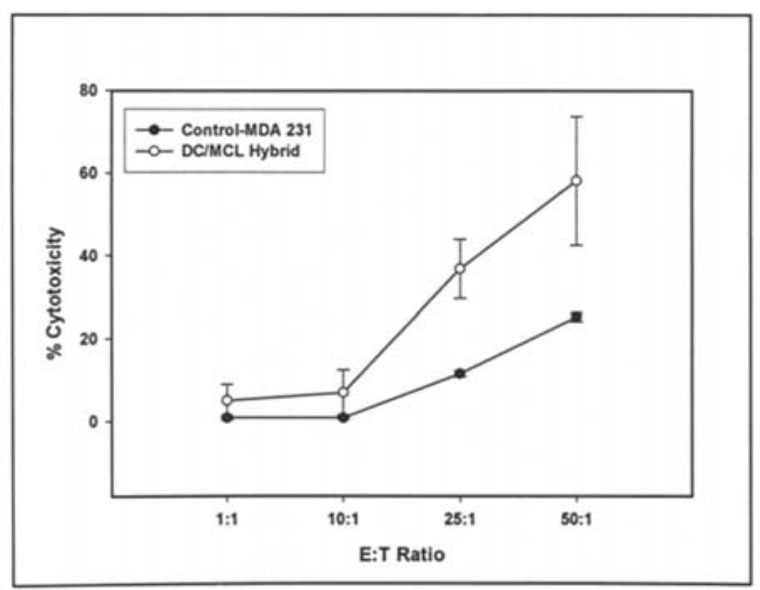

Figure 3. In vitro cytotoxicity levels of MCL-specific cytotoxic T lymphocytes at various effector cell-target cell ratios against Granta 519 MCL target cells. CTLs were generated using T cells stimulated with DCMCL hybrids and tested for their MCL-specific cytotoxicity using in vitro cytotoxicity assay. MDA-231 human breast cancer cells were used as an irrelevant control target for the CTL assay. The values were derived from 4 different experiments.

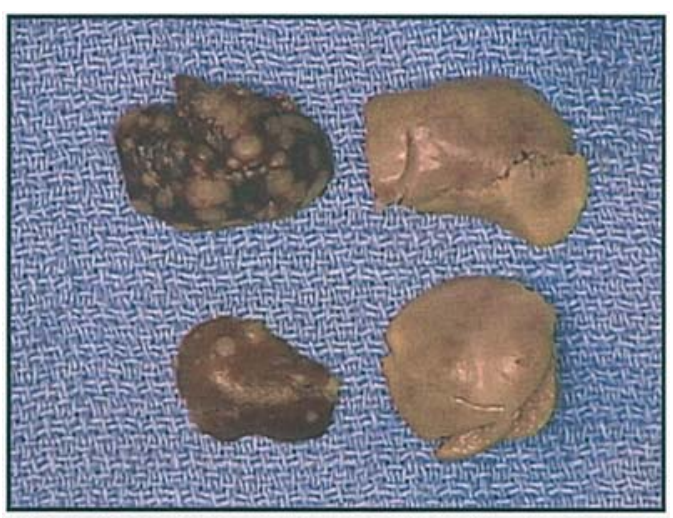

Figure 4. In vivo growth and dissemination of Granta-519 in NOD-SCID mice. Mice were transplanted with ten million Granta-519 MCL cells intravenously and sacrificed after 4 weeks. At the time of necropsy, liver, kidney, spleen and lungs were removed and examined for gross tumor nodules and then processed for histological analyses. Numerous tumor nodules in the livers of the transplanted mice at the time of sacrifice 4 weeks after tumor transplantation was observed, the control livers from mice not transplanted with MCL did not show liver nodules.

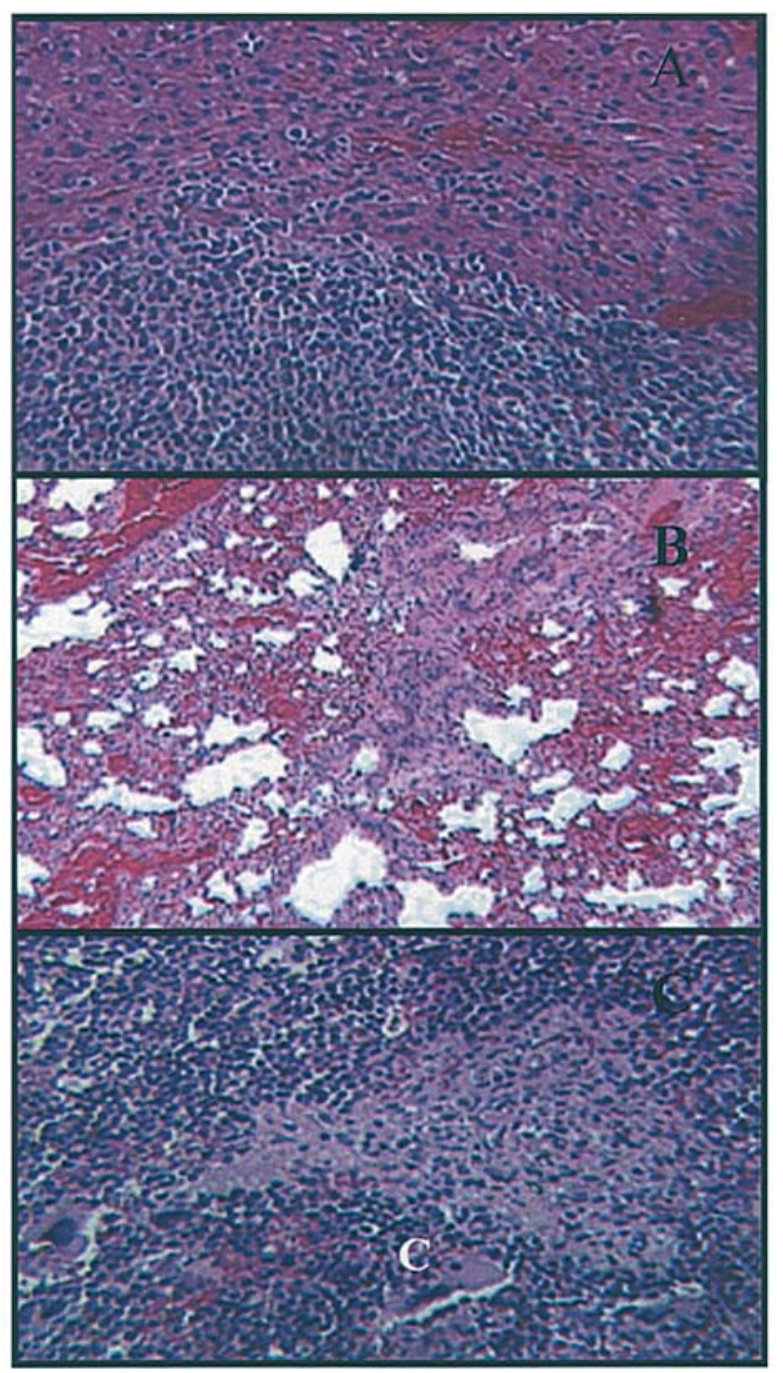

Figure 5. Histological analysis of tissues isolated from NOD-SCID mice transplanted with MCL, (A) liver, (B) lung and (C) spleen.

DC/MCL fusion efficiency. It has been demonstrated that certain cell types can be more or less amenable to cell fusion; thus, it was necessary to calculate the fusion efficiency to determine if sufficient DC/MCL heterokaryons were being produced for use in the in vitro and in vivo experiments. To 


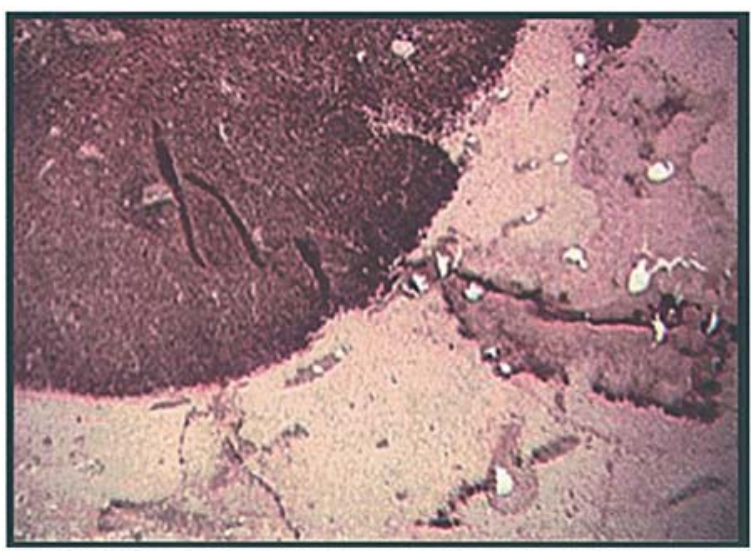

Figure 6. Determination of human origin of the tumors grown in NODSCID mice. Histological section from NOD-SCID mice transplanted with Granta 519 were stained with anti-human CD19 antibody using immunohistochemistry. Tumors in all the organs were positive for anti-human CD19 antibody. A representative micrograph of the liver from one of the tumor bearing-mice is shown.

determine the fusion efficiency of PEG mediated fusion, DCs (red) and Granta 519 (green) cells were stained prior to fusion using CellTracker Orange (CMTMR) and CellTracker Green (CMFDA) dyes as previously described. The fusion efficiency was determined by calculating the percentage of double-stained cells when examined using FACS analysis and confocal microscopy (Fig. 2). The median fusion efficiency was calculated to be in the range of 5-20\%. To eliminate the possibility of the double positive cells being the result of dye leakage and subsequent uptake by the DCs, co-cultures of stained DCs and tumor cells were analyzed as well. The percentages of double-positive cells in the non-fused co-cultures were not significant (data not shown).

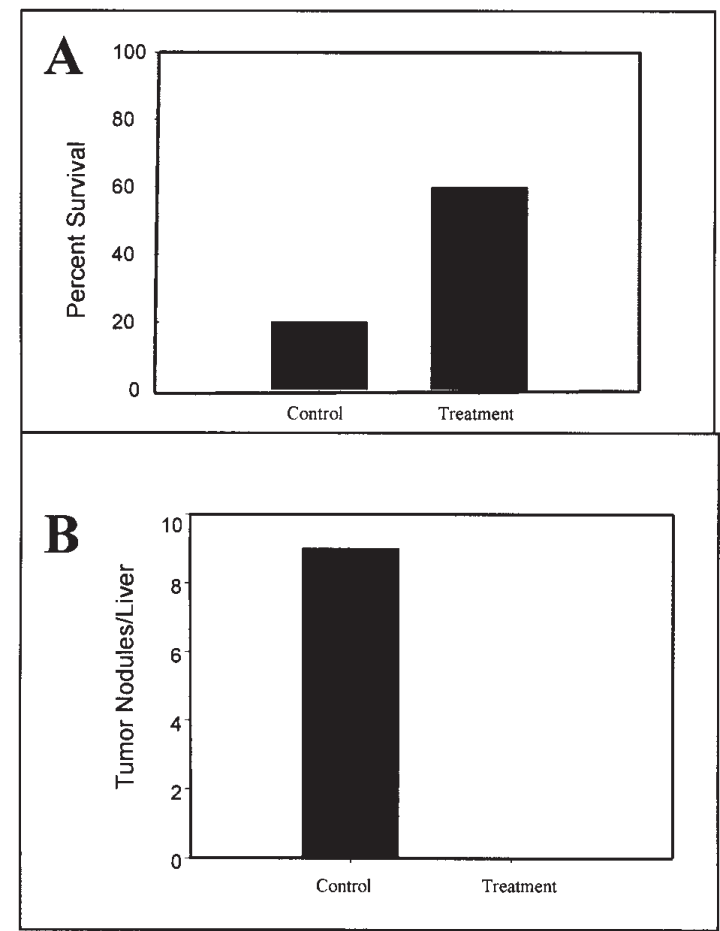

Figure 7. In vivo therapeutic efficacy of DC-MCL-based therapy against MCL Granta 519. (A) Percent survival in the control and treatment groups after 4 weeks of dendritic cell-based immunotherapy $(n=5)$. (B) Average number of tumor nodules per liver in the control and treatment groups. Livers from mice in the treatment group did not contain a noticeable presence of tumor cells.

In vitro cytotoxicity of MCL-specific CTLs against human mantle cell lymphoma. An objective of this study was to compare the immunostimulatory effects among DC-MCL hybrids, for generating MCL-specific CTLs. CTLs were

$A$ and $B$

$\mathrm{B}$ and $\mathrm{C}$

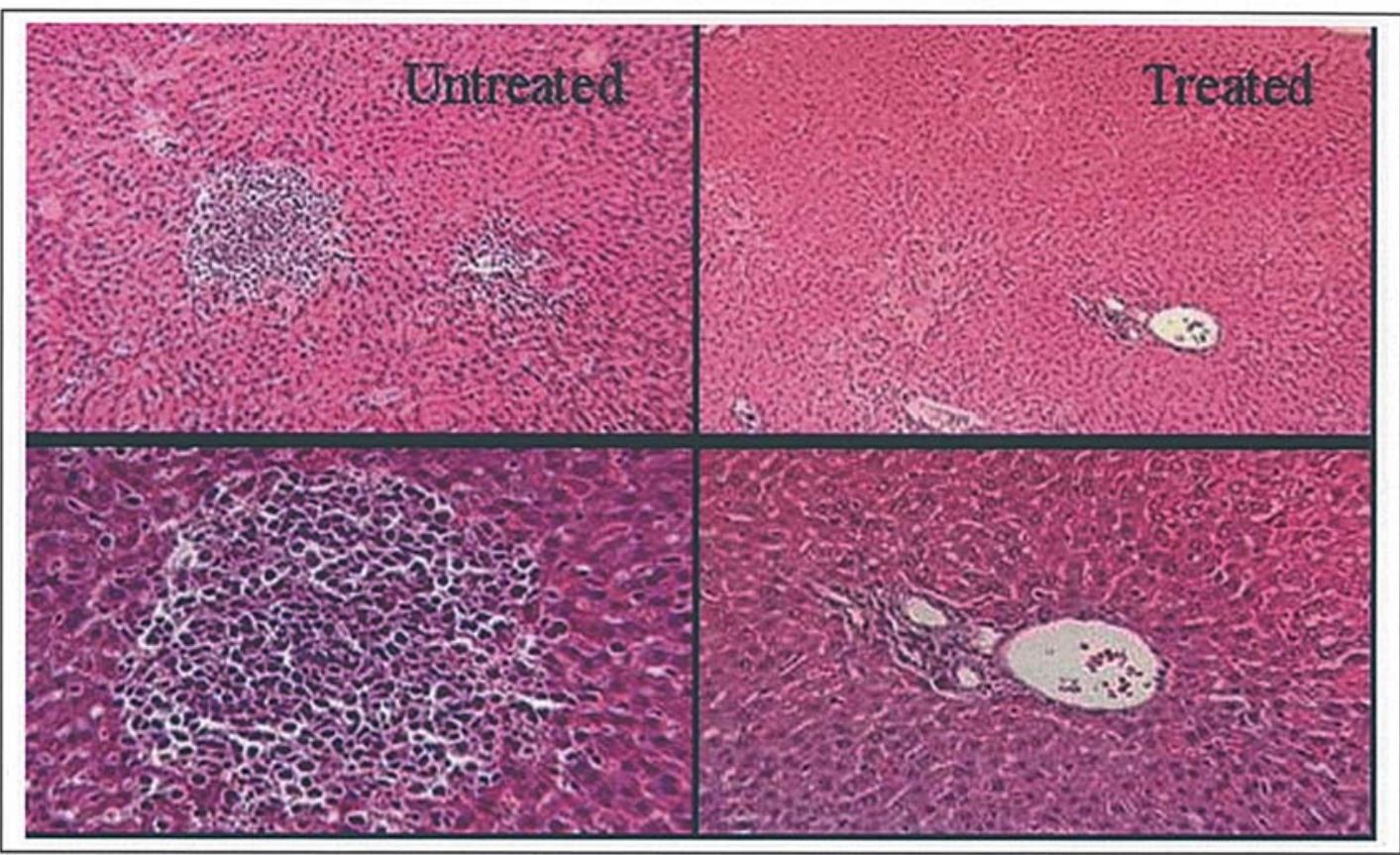

Figure 8. Histological analysis of hepatic tissue from the control and treated mice. (A and B) Tumor-bearing control mice showed tumor nodules in the liver with low and high magnification. (C and D) The tumor nodules were absent in the treated group (right). 
generated using MCL/DC hybrids, with Granta 519 cells as the target cell. The results indicate that DC-MCL-generated CTLs specifically killed Granta-519, but not the irrelevant tumor target IMR-32. Although the lower ET:ratios provided low cytotoxicity, the higher ET:ratios provided $>60 \%$ killing compared to only $20 \%$ for the irrelevant target cells.

Growth of MCL in NOD/SCID mice. For the in vivo studies initially the in vivo model for human MCL cells grown in NOD/SCID mice was developed by transplanting $10 \times 10^{6}$ Granta 519 cells per mouse intravenously. At 3 weeks, the mice were sacrificed and autopsied to examine for the presence of tumor growth and dissemination. Upon visual examination, numerous gross tumor nodules were present on the liver as shown in Fig. 4. Histological analysis of the liver and other tissues revealed tumor infiltration in the spleen, lung, and kidney. Fig. 5 demonstrates the tumor infiltration in liver, lungs and spleen. Immunohistochemical analysis using anti-human CD19 antibody (Fig. 5) was performed to confirm the human origin of the tumor in these organs (Fig. 6). Thus, these results clearly demonstrate that the tumors were of human origin, indicated by the positive staining with human-specific antibody and the Granta-519 grown in NODSCID mouse can be a good preclinical model for in vivo therapeutic studies.

In vivo therapeutic efficacy of DC/MCL hybrid cells. To test the therapeutic immunostimulatory capabilities of DC/MCL hybrid cells against the growth of MCL, NOD/SCID mice bearing MCL were treated once weekly for 4 weeks with DC/MCL hybrids plus IL-2 stimulated cytotoxic T cells. To prevent growth and subsequent GVHD, the effector cells were irradiated prior to administration. Unlike the previous experiment to develop an in vivo model, a low dose $\left(2 \times 10^{5}\right)$ of MCL was transplanted into the mice to better examine the effects of the DC-MCl-based strategy, as the tumor burden would not be as pronounced. After 4 weeks, there was a significant increase in survival of the treated mice as compared to the untreated control mice (Fig. 7). In groups of five mice, three mice in the treatment group remained alive at 4 weeks, as opposed to one mouse in the control group. Histological analysis of liver tissue revealed an average of 9 tumor nodules per liver in the control group, while livers from the surviving mice within the treatment group were free of tumor cells (Figs. 7B and 8). Thus, the in vivo studies demonstrate a therapeutic effect of a DC-based approach against MCL growth in NOD/SCID mice using DC-MCL hybrid cells as immunostimulator for CTL generation.

\section{Discussion}

Relapse in patients with mantle cell lymphoma is caused by the presence of therapy-resistant residual MCL cells. The conventional therapies and host defense mechanism fail to eliminate the residual cells, possibly because the MCL cells may be weakly immunogenic, or the level of immunosuppression within the host prevents the establishment of an effective immune response. To increase the immune response within the host, it may be necessary to introduce antigen-pulsed DCs into the host. This strategy would make use of the antigen presenting capabilities of the DCs to present many MCL associated antigens to the host effector cells in order to stimulate an effective immune response.

The main focus of this study was to determine the immunostimulatory properties possessed by DC/MCL heterokaryons in increasing the effector cell-mediated cytotoxicity toward MCL in vitro and in vivo. Using in vitro CTL cytotoxicity assays, it was found that DC/MCL heterokaryons stimulated CTLs with significant cytotoxicity against Granta 519 cells.

The in vivo arm of this study involved introducing irradiated DC/MCL hybrid cells and T cell-enriched mononuclear cells into NOD/SCID mice transplanted with a low dose of Granta 519 cells. After 4 weekly cellular therapies, $60 \%$ of the mice from the treatment group were alive versus $20 \%$ of the control group. Histological analysis of liver tissue from control and treated groups indicated a significant decrease in the number of tumor nodules within the treatment group. Similar in vivo anti-tumor effects of DC/tumor cell hybrids in mice have been previously reported (28-30). The data clearly demonstrate the therapeutic potential of DC/MCL hybrids to elicit cytotoxic responses in tumorbearing mice. However, it is possible that the observed effects may be due to allo-reactivity against MCL. While this is a possibility, it is likely the observed cytotoxicities, particularly in the mice, are a combination of allogeneic and MCL-specific responses given the data obtained for the in vitro cytotoxicity experiments. The significantly lower cytotoxicity toward the irrelevant neuroblastoma (IMR-32) tumor cells demonstrates the least allogeneic cytotoxicity of this system.

An aim of this study was to develop an immune-based therapy to eliminate residual MCL remaining after initial frontline therapies such as Rituximab and/or combination chemotherapy regimens. Allogenic stem cell transplantation is one such strategy. Allogenic transplantation has been used successfully to induce an immune-mediated graft-versusleukemia (GVL) effect in patients with hematological malignancies. Although the donor $\mathrm{T}$ cells possess the desired GVL effect, unfortunately, these same cells can initiate the potentially lethal graft-versus-host (GVHD) effect as well. For the in vivo portion of this study, irradiation of the effector cells prior to injection was necessary to prevent effector cell proliferation and GVHD within the NOD-SCID mice. While the effector cells will have significantly decreased proliferative potential post irradiation, research suggests these cells still retain CTL capabilities (epitope recognition-cytoxicity) (31). Due to the irradiation, signs of GVHD were not observed in the transplanted NOD/SCID mice.

The data presented here have clearly shown the ability of dendritic cell therapy to increase cytotoxicity toward MCL in vitro and in vivo. The clinical applications for DC therapy are evident, particularly for the treatment of residual lymphoma. It is crucial for the overall survival of patients that residual MCL cells are eliminated. Thus, the antigen presenting properties of dendritic cells make DC therapy a powerful tool to treat patients following cyto-reductive chemotherapy to eliminate residual tumor cells that are resistant to initial or maintenance therapy. It would have been beneficial to have used primary tumor cells isolated from MCL patients in addition to MCL cell line, such as Granta 519. However, recent research indicates that Granta 519 was indeed 
derived from a primary MCL tumor, and continues to express similar genetic and phenotypic characteristics as compared to a primary tumor (32). Such studies using DC-MCL hybrids against primary MCL grown in NOD-SCID mice are in progress. In conclusion, this study details preliminary evidence for the use of DC-based immunotherapy for the effective treatment of mantle cell lymphoma, and lays the foundation to begin additional pre-clinical and clinical studies to evaluate the feasibility and efficacy of this approach in the treatment of MCL patients.

\section{Acknowledgements}

This study was partially supported by the State of Nebraska Department of Health and Human Services, LB506 Funds and Lymphoma Research Foundation, New York, NY.

\section{References}

1. Kauh J, Baidas SM, Ozdemirli M and Cheson BD: Mantle cell lymphoma: clinicopathologic features and treatments. Oncology 17: 879-891, 2003.

2. Decaudin D: Mantle cell lymphoma: a biological and therapeutic paradigm. Leuk Lymphoma 43: 773-781, 2002.

3. Bertoni F, Zucca E and Cavalli F: Mantle cell lymphoma. Curr Opin Hematol 11: 411-418, 2004

4. Lenz G, Dreyling M and Hiddemann W: Mantle cell lymphoma: established therapeutic options and future directions. Ann Hematol 83: 71-77, 2004.

5. Zhu Y, Hollmen J, Katy R, Aalto Y, Nagy B, Elonen E, Kere J, Mannila H, Franssila and Knuutila KS: Investigatory and analytical approaches to differential gene expression profiling in mantle cell lymphoma. Br J Haematol 119: 905-915, 2002.

6. Staudt L, et al: The proliferation gene expression signature is a quantitative integrator of oncogenic events that predicts survival in mantle cell lymphoma. Cancer Cell 3: 185-197, 2003.

7. Gianni A, Magni M, Martelli M, Di Nicola M, Carlo-Stella C, Pilotti S, Rambaldi A, Cortelazzo S, Patti C, Parvis G, Benedetti F, Capria S, Corradini P, Tarella C and Barbui T: Long-term remission in mantle cell lymphoma following high-dose sequential chemotherapy and in vivo rituximab-purged stem cell allografting (R-HDS regimen). Blood 102: 749-755, 2003.

8. Hess G, Flohr T, Huber C, Kolbe K, Derigs H-G and Fischer T: Safety and feasibility of $\mathrm{CHOP} /$ rituximab induction treatment followed by high-dose chemo/radiotherapy and autologous PBSC-transplantation in patients with previously untreated mantle cell or indolent B-cell-non-Hodgkin's lymphoma. Bone Marrow Transplant 31: 775-782, 2003.

9. Andersen N, Pedersen L, Elonen E, Johnson A, Kolstad A, Franssila K, Langholm R, Ralfkiaer E, Akerman M, Eriksson M, Kuittinen $\mathrm{O}$ and Geisler C: Primary treatment with autologous stem cell transplantation in mantle cell lymphoma: outcome related to remission pretransplant. Eur J Haematol 71: 73-80, 2003.

10. Howard O, Gribben J, Neuberg D, Grossbard M, Poor C, Janicek M and Shipp M: Rituximab and CHOP induction therapy for newly diagnosed mantle-cell lymphoma: molecular complete responses are not predictive of progression-free survival. J Clin Oncol 20: 1288-1294, 2002.

11. Lazzarino M, Arcaini L, Bernasconi P, Alessandrino E, Gargantini L, Cairoli R, Orlandi E, Astori C, Brusamolino E, Pagnucco G, Colombo A, Caltroni S, Iacona I, Regazzi M and Morra E: A sequence of immuno-chemotherapy with Rituximab, mobilization of in vivo purged stem cells, high-dose chemotherapy and autotransplant is an effective and non-toxic treatment for advanced follicular and mantle cell lymphoma. $\mathrm{Br}$ J Haematol 116: 229-235, 2002.

12. Vandenberghe E, De Elvira C, Loberiza F, Lopez-Guillermo A, Conde E, Gisselbrecht C, Guilhot F, Vose J, van Biesen K, Rizzo J, Weisenburger D, Isaacson P, Horowitz M, Goldstone A, Lazarus $\mathrm{H}$ and Schmitz N: Outcome of autologous transplantation for mantle cell lymphoma: a study by the European Blood and Bone Marrow Transplant and Autologous Blood and Marrow Transplant Registries. Br J Haematol 120: 793-800, 2003.
13. Whiteside $\mathrm{T}$ and Odoux C: Dendritic cell biology and cancer therapy. Cancer Immunol Immunother 53: 240-248, 2004.

14. O'Neill D, Adams S and Bhardwaj N: Manipulating dendritic cell biology for the active immunotherapy of cancer. Blood 104: 2235-2246, 2004.

15. Delamarre L, Holcombe $\mathrm{H}$ and Mellman I: Presentation of exogenous antigens on major histocompatability complex (MHC) class I and MHC class II molecules is differentially regulated during dendritic cell maturation. J Exp Med 198: 111-122, 2003.

16. Shimizu K, Kuriyama H, Kjaergaard J, Lee W, Tanaka H and Shu S: Comparative analysis of antigen loading strategies of dendritic cells for tumor immunotherapy. J Immunother 27: 265-272, 2004.

17. Ribas A, Butterfield L, Glaspy J and Economou J: Current developments on cancer vaccines and cellular immunotherapy. J Clin Oncol 21: 2415-2432, 2003

18. Gottfreid E, Krieg R, Eichelberg C, Andreesen R, Mackensen A and Krause W: Characterization of cells prepared by dendritic cell-tumor cell fusion. Cancer Immunity 2: 15-26, 2002.

19. Scott-Taylor TH, Pettengell R, Clarke I, Stuhler G, La Barthe MC, Walden P and Dalgleish AG: Human tumour and dendritic cell hybrids generated by electrofusion: potential for cancer vaccines. Biochim Biophys Acta 1500: 265-279, 2000.

20. Geiger J, Hutchinson R, Hohenkirk L, McKenna E, Yanik G, Levine J, Chang A, Braun T and Mule J: Vaccination of pediatric solid tumor patients with tumor lysate-pulsed dendritic cells can expand specific $\mathrm{T}$ cells and mediate tumor regression. Cancer Res 61: 8513-8519, 2001

21. Gilboa E and Vieweg J: Cancer immunotherapy with mRNAtransfected dendritic cells. Immunol Rev 199: 251-263, 2004.

22. Milazzo C, Reichardt VL, Muller MR, Grunebach F and Brossart P: Induction of myeloma-specific cytotoxic T cells using dendritic cells transfected with tumor-derived RNA. Blood 101: 977-982, 2003.

23. Busca A, Bruno B, Boccadoro M, Locatelli F and Falda M: Allogenic stem cell transplantation following non-myeloablative conditioning regimens as adoptive immunotherapy in patients with hematological malignancies. Med Sci Monit 8: RA221-RA232, 2002.

24. Hogan WJ and Deeg HJ: Stem cell transplantation: graftmediated antileukemia effects. Methods Mol Med 109: 421-444, 2005.

25. Chakrabarti S and Childs R: Allogeneic immune replacement as cancer immunotherapy. Expert Opin Biol Ther 3: 1051-1060, 2003.

26. Grunebach F, Muller MR, Nencioni A and Brossart P: Delivery of tumor-derived RNA for the induction of cytotoxic Tlymphocytes. Gene Ther 10: 367-374, 2003

27. Wang P, Munger CM, Joshi AD, Pirruccello SJ and Joshi SS: Cytotoxicity of cord blood derived Her2/neu-specific cytotoxic T lymphocytes against human breast cancer in vitro and in vivo. Breast Cancer Res Treat 83: 15-23, 2004.

28. Siders W, Vergilis K, Johnson C, Shields J and Kaplan J: Induction of specific antitumor immunity in the mouse with the electrofusion product of tumor cells and dendritic cells. Mol Ther 7: 498-505, 2003.

29. Takeda A, Homma S, Okamoto T, Kufe D and Ohno T: Immature dendritic cell/tumor cell fusions induce potent antitumor immunity. Eur J Clin Inv 33: 897-904, 2003.

30. Tanaka H, Shimizu K, Hayashi T and Shu S: Theraputic immune response induced by electrofusion of dendritic and tumor cells. Cell Immunol 220: 1-12, 2002.

31. Gowing H, Lawler M, Hagenbeek A, McCann S, Pamphilon D, Hudson J, van Weelden H, Braakman E and Martens C: Effect of ultraviolet-B light on lymphocyte activity and doses which normal bone marrow cells are preserved. Blood 87: 1635-1643, 1996.

32. Ek S, Ortega E and Borrebaeck CA: Transcriptional profiling and assessment of cell lines as in vitro models for mantle cell lymphoma. Leuk Res 29: 205-213, 2005. 